$\mathrm{SPhT} / 95 / 003$

\title{
Exact Solution of Long-Range Interacting Spin Chains with Boundaries
}

\author{
D. Bernard*, V. Pasquier and D. Serban \\ Service de Physique Théorique ${ }^{\dagger}$, \\ CE Saclay, 91191 Gif-sur-Yvette, France
}

\begin{abstract}
We consider integrable models of the Haldane-Shastry type with open boundary conditions. We define monodromy matrices, obeying the reflection equation, which generate the symmetries of these models. Using a map to the Calogero-Sutherland Hamiltonian of $\mathrm{BC}$ type, we derive the spectrum and the highest weight eigenstates.
\end{abstract}

1/95

* Member of the CNRS

$\dagger$ Laboratoire de la Direction des Sciences de la Matière du Commissariat à l'Énergie Atomique 
It has recently been understood that while the long-range interacting spin chains capture some of the essential physical features of the Heisenberg spin chain, exact results can often be obtained more explicitly. In particular, the spectrum can be described in terms of elementary excitations called spinons. The expressions of the wave functions can be derived. In this paper we propose generalizations of the Haldane-Shastry Hamiltonian [1] [2] which describe open spin chains with various kinds of boundary conditions.

\section{The Hamiltonians}

We consider a system of spins on a one-dimensional semi-circular lattice, with an interaction depending on the inverse square of the distance between the spins

$$
H=\sum_{i \neq j=1}^{N}\left[h_{i j}\left(P_{i j}-1\right)+\bar{h}_{i j}\left(\bar{P}_{i j}-1\right)\right]+\sum_{i=1}^{N}\left(b_{1} h_{i 0}+2 b_{2} \bar{h}_{i i}\right)\left(P_{i}-1\right) .
$$

The operators $P_{i j}$ permute the spins at the sites $i$ and $j$. They correspond to a Heisenbergtype interaction, $P_{i j}=\left(\sigma_{i}^{a} \sigma_{j}^{a}+1\right) / 2$, with $\sigma^{a}$ the Pauli matrices. $P_{i}$ is a reflection operator, $P_{i}^{2}=1$, whose representation will be chosen later and $\bar{P}_{i j}=P_{i} P_{j} P_{i j}$. The coupling constants $h_{i j}$ are defined as follows:

$$
h_{i j}=-\frac{z_{i} z_{j}}{\left(z_{i}-z_{j}\right)^{2}}, \quad \bar{h}_{i j}=-\frac{z_{i} z_{j}^{-1}}{\left(z_{i}-z_{j}^{-1}\right)^{2}}, \quad h_{i 0}=-\frac{z_{i}}{\left(z_{i}-1\right)^{2}}
$$

with $z_{i}$ points on the unit circle characterizing the positions of the spins.

There are at least two possible representations for the reflection operators $P_{i}$; the simplest one is to put $P_{i}=1$, which leads to $P_{i j}=\bar{P}_{i j}$. The Hamiltonian (1.1) then becomes

$$
H=\sum_{i \neq j=1}^{N}\left(h_{i j}+\bar{h}_{i j}\right)\left(P_{i j}-1\right) .
$$

This model is $s u(2)$ symmetric. It was first considered by Simons and Altshuler [3].

The reflection operators can be equally represented by the Pauli matrices $P_{i}=\sigma_{i}^{3}$, in which case the $s u(2)$ invariance is lost; only the projection of the total spin is conserved.

These models can be interpreted as open versions of the Haldane-Shastry spin model [1] [2]. The terms involving $h_{i j}$ couple the 'real' spins $i$ and $j$, while the terms with coupling constants $\bar{h}_{i j}$ correspond to the interaction of the spin $i$ with the image of the spin $j$.

When $P_{i}=\sigma_{i}^{3}$, the last two terms in (1.1) can be interpreted as space dependent magnetic fields varying as $1 / \sin ^{2} x$, with $x$ being the angle (or two times the angle) between a spin and the boundary. Remark that for the term $\bar{h}_{i i}$ the two boundaries are equivalent, while for the term $h_{i 0}$ they are not. This term breaks the $z_{i} \leftrightarrow-z_{i}$ invariance. 


\section{Integrability Condition}

This Hamiltonian is integrable only for certain values of the numbers $z_{i}$ and of the parameters $b_{1}, b_{2}$. To obtain the condition of integrability we shall follow the approach of ref. [4]. In addition to the operators which permute the spins, let us introduce permutation and reflection operators acting on the coordinates as

$$
K_{i j} z_{i}=z_{j} K_{i j}, \quad K_{i} z_{i}=z_{i}^{-1} K_{i}, \quad \bar{K}_{i j} z_{i}=z_{j}^{-1} \bar{K}_{i j} .
$$

Introduce also a set of mutually commuting operators (Dunkl operators) [5]

$$
d_{i}=\sum_{j ; j>i} \theta_{i j} K_{i j}-\sum_{j ; j<i} \theta_{j i} K_{i j}+\sum_{i \neq j} \bar{\theta}_{i j} \bar{K}_{i j}+\left(b_{1} \theta_{i 0}+b_{2} \bar{\theta}_{i i}\right) K_{i}
$$

with $\theta_{i j}=z_{i} /\left(z_{i}-z_{j}\right), \bar{\theta}_{i j}=z_{i} /\left(z_{i}-z_{j}^{-1}\right)$ and $\theta_{i 0}=z_{i} /\left(z_{i}-1\right)$. We denote by $\pi$ a projection operation which consists in replacing the coordinate permutations (reflections) by spin permutations (reflections) when they are at the right of an expression. Let us consider the Hamiltonian $\widehat{H}$ with the property $H=\pi(\widehat{H})$

$$
\widehat{H}=\sum_{i \neq j=1}^{N}\left[h_{i j}\left(K_{i j}-1\right)+\bar{h}_{i j}\left(\bar{K}_{i j}-1\right)\right]+\sum_{i=1}^{N}\left(b_{1} h_{i 0}+2 b_{2} \bar{h}_{i i}\right)\left(K_{i}-1\right) .
$$

This Hamiltonian commutes with the reflection and permutation operators, $\left[\widehat{H}, K_{i}\right]=0$, $\left[\widehat{H}, K_{i j}\right]=0$, so its eigenfunctions can be chosen either symmetric or antisymmetric under the reflection $z_{i} \rightarrow z_{i}^{-1}$ and permutation of coordinates. $\widehat{H}$ can be diagonalized on a basis of functions depending on coordinates and spins, $\Psi$. These are also the eigenfunctions of $H$, provided that $\left(K_{i}-P_{i}\right) \Psi=0,\left(K_{i j}-P_{i j}\right) \Psi=0$. Choosing $K_{i}=+1$ or -1 lead to the choice $P_{i}=1$ or $P_{i}=\sigma_{i}^{3}$.

The integrability condition is that the Hamiltonian (2.3) commutes with the Dunkl operators, $\left[\widehat{H}, d_{i}\right]=0$. It can be translated into $N$ equations for the coordinates $z_{i}$ and the constants $b_{1,2}$

$$
2 \sum_{j ; j \neq i}^{N}\left(h_{i j} w_{i j}+\bar{h}_{i j} \bar{w}_{i j}\right)+2 b_{2}^{2} \bar{h}_{i i} \bar{w}_{i i}+b_{1}\left(b_{1}+b_{2}\right) h_{i 0} w_{i 0}=0
$$

where $w_{i j}=\left(z_{i}+z_{j}\right) /\left(z_{i}-z_{j}\right), \bar{w}_{i j}=\left(z_{i}+z_{j}^{-1}\right) /\left(z_{i}-z_{j}^{-1}\right)$ and $w_{i 0}=\left(z_{i}+1\right) /\left(z_{i}-1\right)$. The system of equations $\sum_{j ; j \neq i}^{L} h_{i j} w_{i j}=0$ has the solution $z_{k}=C \mathrm{e}^{2 i \pi k / L}$ with $C$ a multiplicative constant. We derive solutions for the equations (2.4) by putting them in 


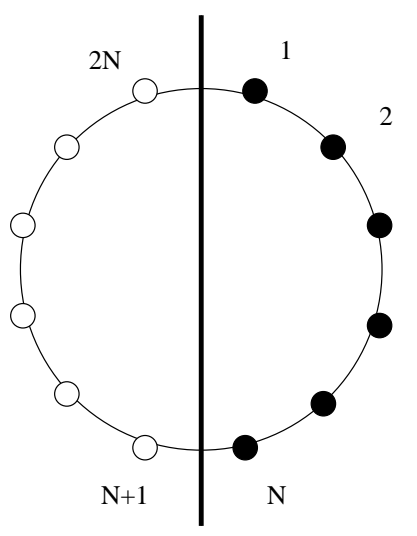

i) $\mathrm{L}=2 \mathrm{~N}$

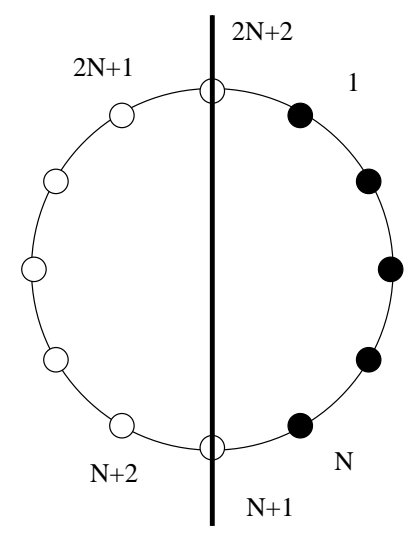

ii) $\mathrm{L}=2 \mathrm{~N}+2$

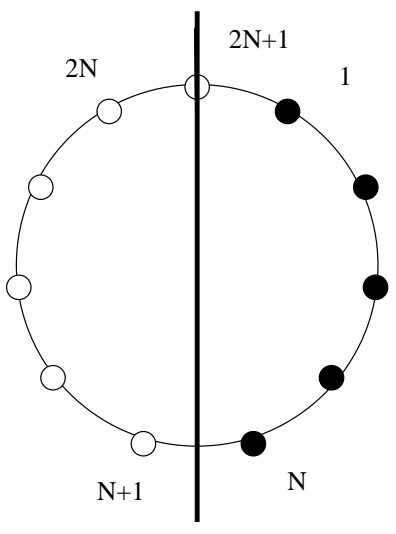

iii) $\mathrm{L}=2 \mathrm{~N}+1$

Fig. 1: Distribution of the spins on the lattice. The bold line represents the boundary (mirror), the filled circles indicate the positions of the spins. The open dots are the images of the 'physical' points through the mirror.

this form. Different solutions correspond to different distributions of the coordinates $z_{i}$, as illustrated in Fig. 1.

We consider a circular lattice with $L$ sites and set $\omega=\mathrm{e}^{2 i \pi / L}$ :

i) If the 'mirror' (the boundary) is placed between the sites of the lattice, then $L=2 \mathrm{~N}$, $N$ being the number of 'physical' sites. In this case, $z_{k}=\omega^{k-1 / 2}$ and the constants in (1.1) have the values $b_{1}=0, b_{2}= \pm 1$.

ii) If the mirror passes through the sites of the lattice, $L=2 N+2$ and $z_{k}=\omega^{k}$. The constants in the Hamiltonian have the values $b_{1}=0, b_{2}= \pm 3$.

iii) The third case corresponds to a mirror passing through a site and a bond, so $L$ is odd, $L=2 N+1$. The positions of the sites are $z_{k}=\omega^{k}$ and $b_{2}= \pm 1$. The constant $b_{1}$ acquires a non-zero value and it is the solution of the equation $b_{1}\left(b_{1}+b_{2}\right)=2$.

When the reflection operator is represented by the identity, the constants $b_{1}$ and $b_{2}$ are not relevant, as they multiply constant terms in (1.1). When the reflection is represented by $\sigma_{i}^{3}$, an overall change of sign of the constants $b_{1}, b_{2}$ corresponds to the conjugation $\sigma_{i}^{3} \rightarrow-\sigma_{i}^{3}, \sigma_{i}^{ \pm} \rightarrow \sigma_{i}^{\mp}$. In this case we will retain only the positive value of $b_{2}$.

\section{Symmetry and Spectrum}

The model possesses a monodromy matrix which commutes with the Hamiltonian $H$ and which satisfies the reflection equation [6] :

$$
R_{00^{\prime}}(u-v) T^{0}(u) \bar{R}_{00^{\prime}}(u+v) T^{0^{\prime}}(v)=T^{0^{\prime}}(v) \bar{R}_{00^{\prime}}(u+v) T^{0}(u) R_{00^{\prime}}(u-v)
$$


where $T^{0}(u)$ stands for $T(u) \otimes 1$ and $T^{0^{\prime}}(u)$ for $1 \otimes T(u)$. The matrices $R(u)$ and $\bar{R}(u)$ are given by :

$$
R(u)=u+P_{00^{\prime}}, \quad \bar{R}(u)=u+\bar{P}_{00^{\prime}}
$$

where the operator $P_{00^{\prime}}$ permutes the two auxiliary spaces 0 and $0^{\prime}$ and $\bar{P}_{00^{\prime}}$ represents permutation followed by a reflection in each space. The explicit expression of this monodromy matrix is

$$
T(u)=\pi\left[\prod_{i=1}^{N}\left(1+\frac{P_{i 0}}{u-d_{i}}\right)\left(1+\frac{b_{1}+b_{2}}{2} \frac{P_{0}}{u}\right) \prod_{i=N}^{1}\left(1+\frac{\bar{P}_{i 0}}{u+d_{i}}\right)\right] .
$$

Expanding $T(u)$ in powers of $u^{-1}$ around the point at the infinity generates conserved charges, $T(u)=I+\sum_{n \geq 0} T_{n} u^{-(n+1)}$. If $P_{i}=1$, the first conserved charge is the total spin $Q_{0}^{a}=\sum_{i=1}^{N} \sigma_{i}^{a}$ and the non-trivial charges are located at even levels, $Q_{2 n}$. If $P_{i}=\sigma_{i}^{3}$, the conserved charge at the level zero is the projection of the total spin, $Q_{0}^{3}=\sum_{i=1}^{N} \sigma_{i}^{3}$. The $s u(2)$ invariance is broken by the presence of the last two terms in the Hamiltonian (1.1). At the next level, we find two non-trivial generators :

$$
Q_{1}^{ \pm}=\frac{1}{2} \sum_{i=1}^{N} \sigma_{i}^{ \pm}\left[\sum_{j ; j \neq i}\left(w_{i j} P_{i j}+\bar{w}_{i j} \bar{P}_{i j}\right)+b_{1} w_{i 0} P_{i}+b_{2} \bar{w}_{i i} P_{i}\right] .
$$

As a consequence of these symmetries, the energy levels are degenerate and the eigenvectors are grouped in multiplets. Each multiplet is characterized by a set of rapidities $\left\{m_{i}\right\}, c_{1} / 2+c_{2} \leq m_{i} \leq L / 2-1$, where $c_{1}, c_{2}$ and $L / 2-m_{i}$ are integers. The values of $c_{1}$ and $c_{2}$ corresponding to the different cases are summarized below

\begin{tabular}{|l|l|cc|rccc|}
\hline & & \multicolumn{2}{|c|}{$P_{i}=1$} & $P_{i}$ & \multicolumn{2}{c|}{$\sigma_{i}^{3}$} \\
\hline$L$ & $m_{i}$ & $c_{1}$ & $c_{2}$ & $b_{1}$ & $b_{2}$ & $c_{1}$ & $c_{2}$ \\
\hline $2 N$ & $Z$ & 0 & 1 & 0 & 1 & 0 & 0 \\
$2 N+2$ & $Z$ & 0 & 2 & 0 & 3 & 0 & 1 \\
$2 N+1$ & $Z+1 / 2$ & 1 & 1 & 1 & 1 & 1 & 0 \\
& & & & -2 & 1 & 3 & 0 \\
\hline
\end{tabular}

Table 1.

The energy of a multiplet have the expression

$$
E_{\left\{m_{i}\right\}}=\sum_{j=1}^{M}\left(m_{j}^{2}-\frac{L^{2}}{4}\right)
$$


The form of the spectrum will be justified later, using a map to a continuous model, the Calogero-Sutherland model.

The rules giving the degeneracies of the spectrum depend on the symmetry of the model, hence on the representation chosen for the operators $P_{i}$. They have been determined numerically and they should also follow from a careful analysis of the representation of the symmetry algebra (3.1). If $P_{i}=1$, the model possesses a $s u(2)$ symmetry and the degeneracies are the same as for the Haldane-Shastry model [7]. The only difference is that the energy is not invariant under the transformation $m_{i} \rightarrow N-m_{i}$. The rule can be formulated as follows: take a chain of $N$ fictitious spins with values + and - (these are not the spins in (1.1)). Put a 1 between two consecutive fictitious spins if the first one is greater than the second and a 0 otherwise. This sequence of 0 and 1 is called a 'motif' and the positions on the motif are labeled by integers (half-integers) ranging from $c_{1} / 2+c_{2}$ to $L / 2-1$. The positions of the 1's give the set of quantum numbers $\left\{m_{i}\right\}$. The degeneracy associated to a given motif equals the number of configurations of the fictitious spins compatible with it.

If $P_{i}=\sigma_{i}^{3}$, the symmetry group is $u(1)$ and we expect the spectrum to be less degenerate. In this case, the rule giving the degeneracies is slightly modified from the one above. We must consider now a chain of $N+2$ fictitious spins, with the first and the last ones fixed at the value + . This constraint imposes to all the fictitious spins at the left of the first 1 to have the value + . The positions of the symbols on the motif range from $c_{1} / 2+c_{2}$ to $L / 2$. For the case $L=2 N+1, b_{1}=-2$ we obtained numerical evidence for a $s u(2)$ symmetry, but we did not succeed to find the conserved generators (which are not the total spin operators). In this case, the degeneracies are the same as for the periodic chain.

\section{Mapping to the Calogero-Sutherand Model}

The eigenvectors and the eigenvalues of the Hamiltonian (1.1) can be obtained from a correspondence with a particular model of Calogero-Sutherland (CS) type. Before giving the details of the correspondence, let us give the definition of the general CS model. This model describes $n$ particles on a line, with coordinates $x_{i}, 0 \leq x_{i} \leq l$. In the version proposed by Calogero and Sutherland [8] [9], the interaction among the particles is pairwise and the model is completely integrable. In fact, the model is integrable for more general potentials, associated to all the root systems of the simple Lie algebras [10]. 
Let $V$ denote a $n$ dimensional vector space with an orthonormal basis $\left\{e_{1}, \ldots, e_{n}\right\}$ and let $R=\{\alpha\}$ be a root system spanning $V$, with $R_{+}$the set of positive roots. Let $x$ denote the vector $\left(x_{1}, \ldots, x_{n}\right)$ and $x \cdot \alpha$ its scalar product with the vector $\alpha$. Then the general CS Hamiltonian is

$$
H_{C S}=-\sum_{i=1}^{n} \frac{\partial^{2}}{\partial x_{i}^{2}}+\sum_{\alpha \in R_{+}} \frac{g_{\alpha}}{\sin ^{2}(x \cdot \alpha)}
$$

where $g_{\alpha}$ is constant on each orbit of the Weyl group (it has the same value for the roots of the same length). The Hamiltonian proposed by Sutherland and used to solve the Haldane-Shastry model correspond to the root system of type $A_{n}, R=\left\{e_{i}-e_{j}, i \neq j\right\}$. The one relevant for our problem is associated to the $B C_{n}$ (generalized) root system, $R=\left\{ \pm e_{i}, \pm 2 e_{i}, \pm e_{i} \pm e_{j}\right\}$. In this case, the Weyl group is generated by the $n$ reflections on the hyperplanes $x_{i}=0\left(K_{i}\right)$ and by the reflections on the hyperplanes $x_{i}= \pm x_{j}$ (the permutations $K_{i j}, \bar{K}_{i j}$ ).

With the variables $z_{k}=\mathrm{e}^{i x_{k}}$ the Hamiltonian (4.1) becomes:

$$
H_{C S}=\sum_{i=1}^{n}\left(z_{i} \partial_{z_{i}}\right)^{2}+\beta(\beta-1) \sum_{i \neq j=1}^{n}\left(h_{i j}+\bar{h}_{i j}\right)+\sum_{i=1}^{n}\left(c_{1}\left(2 c_{2}+c_{1}-1\right) h_{i 0}+4 c_{2}\left(c_{2}-1\right) \bar{h}_{i i}\right) .
$$

with $h_{i j}$ defined in terms of $z$ as in (1.2). The form of the three independent coupling constants $g_{\alpha}$ was chosen for later convenience. The ground state wave function of this Hamiltonian is

$$
\phi_{0}(z)=\prod_{i} z_{i}^{-\left(\beta(n-1)+c_{2}+c_{1} / 2\right)}\left(z_{i}-1\right)^{c_{1}}\left(z_{i}^{2}-1\right)^{c_{2}} \prod_{i \leq j}\left(z_{i}-z_{j}\right)^{\beta}\left(z_{i} z_{j}-1\right)^{\beta}
$$

In some situations, a gauge transformed version of (4.2), $\widetilde{H}_{C S}=\phi_{0}^{-1} H_{C S} \phi_{0}$, can be useful

$$
\widetilde{H}_{C S}=\sum_{i=1}^{n}\left(z_{i} \partial_{z_{i}}\right)^{2}+\beta \sum_{i \neq j}\left(w_{i j}+\bar{w}_{i j}\right) z_{i} \partial_{z_{i}}+\sum_{i=1}^{n}\left(c_{1} w_{i 0}+2 c_{2} \bar{w}_{i i}\right) z_{i} \partial_{z_{i}}+E_{0}
$$

with $E_{0}=\sum_{i-1}^{n}\left[c_{1} / 2+c_{2}+\beta(n-i)\right]^{2}$. As in the case initially studied by Sutherland, this Hamiltonian is triangular in a basis of symmetrized plane waves

$$
m_{\lambda}=\sum z_{1}^{\lambda_{1}} z_{2}^{\lambda_{2}} \ldots z_{n}^{\lambda_{n}}
$$

where the sum is now over all the permutations and the changes of sign of the integers $\lambda_{i}$ $\left(\lambda_{1} \geq \ldots \geq \lambda_{n} \geq 0\right)$. 
Therefore, the eigenfunctions of the Hamiltonian $H_{C S}$ can be taken of the form

$$
\phi(z)=\phi_{0}(z) P_{\lambda_{1}, \ldots \lambda_{n}}(z)
$$

where the $P(z)$ are polynomials in the variables $z_{i}, z_{i}^{-1}$, symmetric under the permutations $z_{i} \leftrightarrow z_{j}$ and under the reflections $z_{i} \rightarrow z_{i}^{-1}$. Some properties of these polynomials associated to general root systems are studied by Macdonald [11]. The corresponding eigenvalues are

$$
E_{C S}=\sum_{i=1}^{n}\left(\lambda_{i}+\beta(n-i)+c_{1} / 2+c_{2}\right)^{2}
$$

The spectrum and the eigenvectors of the spin chain can be derived from the spectrum and the wave functions of the CS model. We construct the Hilbert space of the chain from the ferromagnetic state $|\Omega\rangle=|++\ldots+\rangle$ by reversing $M$ spins :

$$
|\Psi\rangle=\sum_{n_{1}, \ldots, n_{M}=1}^{N} \psi_{n_{1}, \ldots, n_{M}} \sigma_{n_{1}}^{-} \ldots \sigma_{n_{M}}^{-}|\Omega\rangle
$$

The coefficients $\psi_{n_{1}, \ldots, n_{M}}$ are symmetric under the permutation of their indices. We extend their definition for $1 \leq n \leq L$ by putting $\psi_{\ldots, L-n_{i}+2 \alpha, \ldots}=K_{i} \psi_{\ldots, n_{i}, \ldots}= \pm \psi_{\ldots, n_{i}, \ldots}$ with $\alpha=1 / 2$ if $L=2 N$ and $\alpha=0$ otherwise. The + sign corresponds to the choice $P_{i}=1$ and the - sign to $P_{i}=\sigma_{i}^{3}$. By convention, we chose the coefficients equal to zero if an index equals $L$ or if two indices coincide.

Let now $\Psi\left(z_{1}, \ldots, z_{M}\right)$ be a symmetric polynomial of degree at most $L-1$ in each $z_{i}$ such that $\psi_{n_{1}, \ldots, n_{M}}=\Psi\left(\omega^{n_{1}-\alpha}, \ldots, \omega^{n_{M}-\alpha}\right)$. The condition of antisymmetry of $\psi$ (when $P_{i}=\sigma_{i}^{3}$ ) and the condition for $|\Psi\rangle$ to be a $s u(2)$ highest weight, $\sum_{i=1}^{N} \sigma_{i}^{+}|\Psi\rangle=0$, (when $\left.P_{i}=1\right)$ impose that $\sum_{n_{1}=1}^{L} \psi_{n_{1}, \ldots, n_{M}}=0$. This means that $\Psi\left(z_{1}=0, z_{2}, \ldots, z_{M}\right)=0$ and the degree of $\Psi(z)$ in each variable is greater than 0 .

We want to translate the action of the spin Hamiltonian on the vectors $|\Psi\rangle$ into an action on the functions $\Psi(z)$. To do that, we use the fact that in the basis of polynomials $Q_{k}$ in one variable of degree $(L-1)$ specified by $Q_{k}\left(\omega^{n-\alpha}\right)=\delta_{k}^{n}$, the matrix elements of the derivatives are:

$$
\begin{aligned}
& \left(z \partial_{z}-\frac{L-1}{2}\right) Q_{k}(z)=-\sum_{j ; j \neq k}^{L} \frac{\omega^{k}}{\omega^{k}-\omega^{j}} Q_{j}(z) \\
& z \partial_{z}\left(z \partial_{z}-L\right) Q_{k}(z)=-2 \sum_{j ; j \neq k}^{L} \frac{\omega^{k} \omega^{j}}{\left(\omega^{k}-\omega^{j}\right)^{2}}\left(Q_{j}(z)-Q_{k}(z)\right)
\end{aligned}
$$


This enables to recover the action of the Calogero-Sutherland Hamiltonian on the functions $\phi(z)=\prod_{i=1}^{M} z_{i}^{-L / 2} \Psi(z)$ :

$$
H|\Psi\rangle=\sum_{n_{1}, \ldots, n_{M}}(-1)^{\sum n_{i}}\left[\left(H_{C S}-\frac{M L^{2}}{4}\right) \phi\right]\left(\omega^{n_{1}-\alpha}, \ldots, \omega^{n_{M}-\alpha}\right) \sigma_{n_{1}}^{-}, \ldots, \sigma_{n_{M}}^{-}|\Omega\rangle
$$

The values of the coupling constants $c_{1,2}$ in $H_{C S}$ are the values given in Table 1 and the constant $\beta$ is equal to 2 . Note that, for these values, the energy in (4.7) is consistent with (3.5). The rapidities $m_{i}$ are defined in terms of the integers $\lambda_{i}$ as $m_{i}=\lambda_{i}+2(M-i)+$ $c_{1} / 2+c_{2}$. They are integers or half-integers, depending on the parity of $c_{1}$, and they obey the following selection rules: $m_{i}-m_{i+1} \geq 2$ and $c_{1} / 2+c_{2} \leq m_{i} \leq L / 2-1$. The bounds on the $m_{i}$ follow from the fact that $\Psi(z)$ is a polynomial of degree between 1 and $L-1$ in each variable $z_{i}$.

Thus, we have obtained a set of eigenfunctions of the spin chain from the wave functions (4.6) of the Calogero-Sutherland model. This procedure gives only the highest weight vector (annihilated by $Q_{0}^{+}$or $Q_{1}^{+}$) in each multiplet. The other vectors in the multiplet can be obtained by repeated action of the generators $Q_{0,1}^{a}$.

\section{Acknowledgment}

We wish to thank S.K. Yang for informing us about the reference [3]. 


\section{References}

[1] F.D.M. Haldane, Phys.Rev.Lett. 60 (1988) 635.

[2] B.S. Shastry, Phys.Rev.Lett. 60, 639 (1988).

[3] B.D. Simons, B.L. Altshuler, Phys.Rev. B 50, 1102 (1994).

[4] D.Bernard, M.Gaudin, F.D.M.Haldane and V.Pasquier, J.Phys.A 26 (1993) 5219.

[5] V. Pasquier, in preparation.

[6] E.K. Sklyanin, J.Phys.A Math.Gen 21 (1988) 2375.

[7] F.D.M. Haldane, Z.N.C. Ha, J.C. Talstra, D. Bernard and V. Pasquier, Phys.Rev.Lett. 69, 2021 (1992).

[8] F. Calogero, J. Math. Phys. 10, 2191, (1969).

[9] B. Sutherland, J. Math. Phys. 12, 246 (1971); 12, 251 (1971).

[10] M.A. Olshanetsky, A.M. Perelomov, Phys.Rep. 94 (1983) 313.

[11] I.G. Macdonald, Orthogonal polynomial associated with root systems, preprint (1988). 